\title{
Proposal of the Thin Film Pirani Vacuum Sensor Still Sensitive Above 1 Atmosphere
}

\author{
Takashima Noriaki and Kimura Mitsuteru \\ Faculty of Engineering, Tohoku Gakuin University \\ 13-1, Chuo-1, Tagajo, Miyagi, 985-8537, JAPAN \\ kimura@tjcc.tohoku-gakuin.ac.jp
}

\begin{abstract}
We have extended measurable pressure range of the thin film Pirani vacuum sensor that is still sensitive above 1 atmosphere (atm). In our thin film Pirani vacuum sensor, our proposed temperature difference sensor of the short circuit Seebeck-current detection type thermocouple is used in order to get extremely high sensitivity, especially in high vacuum range. A little temperature difference, which should be zero under very high vacuum, between the microheater and the thermally isolated heading area from the microheater of the cantilever can be measured. We have achieved much wider measurable pressure range over 8 digits by use of our new simple thin film Pirani vacuum sensor than that of the traditional one. We could expand the pressure sensitivity beyond $1 \mathrm{~atm}$. by adoption of the vibration of the sensing cantilever due to the sudden heating.
\end{abstract}

\section{INTRODUCTION}

Principle of the thermal vacuum sensor, such as Pirani vacuum sensor, is based on the dependence of the thermal conductivity and convection of the ambient gas on the vacuum pressure, namely based on measuring the heat loss of the hot object [1].

Author (M. Kimura) has proposed a micro air-bridge heater and suggested that this micro air-bridge heater with a temperature sensor will be used as a flow sensor, thermal vacuum sensor, etc. to improve their sensitivity due to larger interaction area with the air and the hot suspended film [2].

Efforts on improvement of the sensitivity of the thermal vacuum sensor, such as Pirani vacuum sensor, by use of the hot floating membrane and thermopile as a temperature sensor instead of the hot wire have been reported [3].

We have reported on the thin film vacuum sensor with three pn junction diodes as a high sensitive temperature sensor working like a thermistor (we call this "diode thermistor" [4]) and a micro air-bridge heater.

This sensor has about two order or wider sensitivity $\left(2 \times 10^{-3}-1 \times 10^{5} \mathrm{~Pa}\right)$ than the traditional thermal vacuum sensor, such as Pirani vacuum sensor, and has very fast response and very low power consumption [5]. However, we have noticed that it is hard to compensate the effects caused by the ambient temperature change, because we have used the high sensitive absolute temperature sensors of diode-thermistor.

In this paper we have proposed the new type thin film vacuum sensor that has a cantilever structure with new temperature difference sensors of the short circuit Seebeck-current detection type thermocouple, which is proposed by us [8], in order to get higher sensitivity in the higher vacuum range. Temperature difference, which should be zero under the higher vacuum, between microheater and thermally isolated heading area from the microheater is measured under a certain pressure. Even a little temperature difference can be measured in very lower pressure range by the signal amplification.

\section{Principle of the Short Circuit Seebeck-Current Type Thermocouple}

It is well known that the Seebeck coefficient $\alpha_{s}$ for silicon is approximated as a function of electrical resistivity $\rho$ as shown below [6]:

$$
\alpha_{s}=\frac{m k}{q} \ln \frac{\rho}{\rho_{0}} \cdots
$$

, where $\rho_{0} \approx 5 \times 10^{-4} \Omega \mathrm{cm}, \mathrm{m}=2.6$ for silicon (Si), $\mathrm{k}$ is the Boltzmann constant, and $\mathrm{q}$ is the electron charge.

We have noticed that it is easy to decrease the resistivity three or four orders, which can extremely increase the short-circuit Seebeck current of the thermocouple, while the Seebeck coefficient $\alpha_{s}$ decreases only several times [7]. If we adopt the method of the short-circuit Seebeck current measurement using a single thermocouple with the $n^{++}-\mathrm{Si}$ and metal film in stead of the method of the 
open-circuit voltage measurement using the traditional thermopile, it is expected that our proposed single thermocouple with extremely low resistance can generate higher thermoelectric power $W_{i}$ than $W_{v}$ of a traditional open-circuit thermopile, where subscript $\mathrm{i}$ and $\mathrm{v}$ correspond to current measurement (single thermocouple) and voltage measurement (traditional thermopile), respectively.

We can use the imaginary short of the OP amplifier to measure the short-circuit Seebeck current of the single thermocouple sensor as shown in Fig. 1. The combination of the single thermocouple sensor and the OP amplifier will compose temperature difference sensor.

Since the Seebeck voltage $V_{t}$ is given by the following equation,

$V_{t}=\alpha_{s} \Delta T, \cdots \cdot(2)$

,where $\Delta T$ is the temperature difference between the hot junction and the cold one of the thin film thermocouple. The output voltage $\mathrm{V}_{\mathrm{o}}$ of the OP amplifier shown in Fig. 1 is given as,

$$
V_{o}=R_{f} I_{s}=R_{f} V_{t} / r \cdots \cdots
$$

, where $I_{s}$ is the short circuit Seebeck -current, $R_{f}$ the feedback resistance of the OP amplifier circuit and $r$ the internal resistance of the thin film thermocouple. So we can get the temperature difference $\Delta T$ as follows,

$$
\Delta T=V_{o} r / \alpha_{s} R_{f} \cdots \cdot(4)
$$

If we measure the internal resistance of the thin film thermocouple beforehand, we can see the temperature difference $\Delta T$ from the above equation (4). We have measured the Seebeck coefficient $\alpha_{s}$ of the phosphorus diffused Si layer $\left(\mathrm{n}^{++}-\mathrm{Si}\right)$ at $1100^{\circ} \mathrm{C}$ for $2 \mathrm{hrs}$, and found that it is about $-160 \mu \mathrm{V} / \mathrm{K}$.

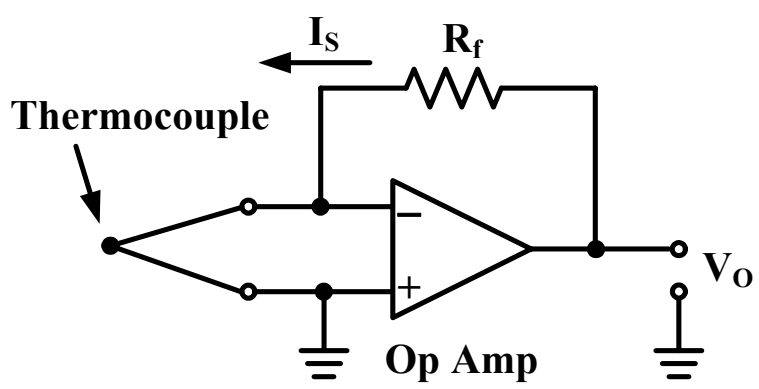

Fig. 1: Measuring circuit for short-circuit current of a single thermocouple using imaginary short of an OP amplifier.

\section{EXPERIMENTS}

\section{Structure and Fabrication Process of the New Type Thin Film Vacuum Sensor}

We have planned to adopt the temperature difference sensor in stead of the absolute temperature sensors such as diode-thermistor in order to get the higher sensitivity in the higher vacuum range. Our proposed short circuit Seebeck-current detection type thermocouple has more merits than the traditional thermopile due to easy fabrication, compactness and sensitivity.

In Fig. 2 the schematic view of the new type thin film vacuum sensor composed of the microheater and two temperature difference sensors of the short circuit Seebeck-current detection type thermocouple formed on the SOI cantilever is shown. The microheater is made of the sputtering deposited Nichrome film and the thin film thermocouple is composed of the phosphorus diffused $\mathrm{Si}$ layer $\left(\mathrm{n}^{++}-\mathrm{Si}\right)$ and $\mathrm{Ni} /$ Nichrome double layer.

In Fig. 3 the micrograph of the sensing region, which consists of the cantilever with two thin film thermocouples, of the new type thin film vacuum sensor is shown.

In Fig. 4 the cross sectional view at X-X' line shown in Fig. 3 on the fabrication process of the new type thin film vacuum sensor is shown.

We have used the $\mathrm{n}-\mathrm{SOI}$ substrate $((100), 7.8 / 1.0 / 550 \mu \mathrm{m}, 3.00-5.00 / 40.0 \Omega \mathrm{cm})$ as a starting material. After initial cleaning, the cantilever pattern is formed by chemical etching of the SOI layer and the thermal silicon dioxide film $\left(\mathrm{SiO}_{2}\right.$ film) is formed by thermal oxidation (Fig.4, step 1). After the lithography of thermal silicon dioxide film (about $0.5 \mu \mathrm{m}$ thick) to open windows for the low resistivity (heavily doped) $\mathrm{n}^{++}$Si regions $\left(<10^{-3} \Omega \mathrm{cm}\right.$ ), thermal diffusion using phosphorous $O C D$-coating diffusion source at $1100^{\circ} \mathrm{C}$ for 1 hour is carried out. Then the thermal diffusion for $\mathrm{p}^{+}$region formation of pn diode is carried out using the 


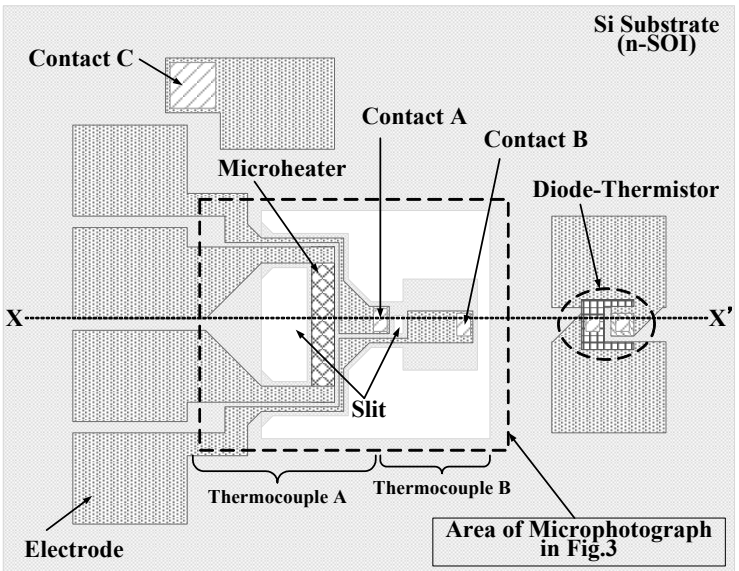

Fig. 2: Schematic view of the new type thin film vacuum sensor.

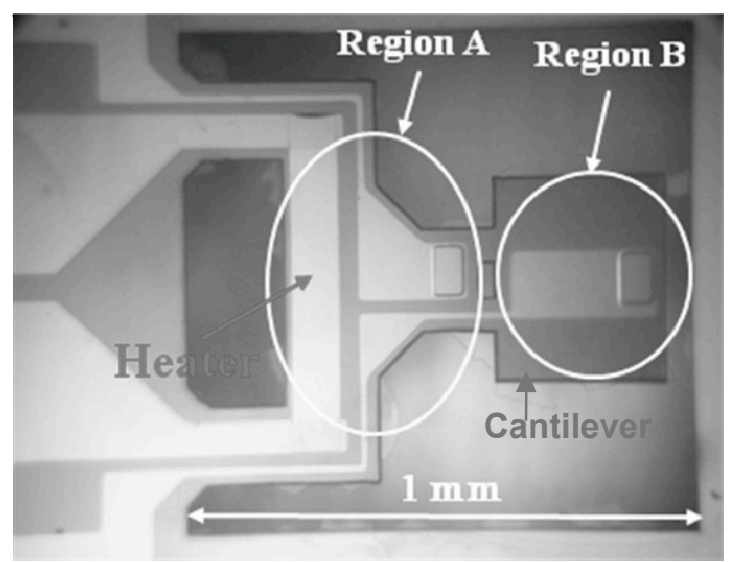

Fig.3: Micrograph of the sensing region of the new type thin film vacuum sensor.
(A)

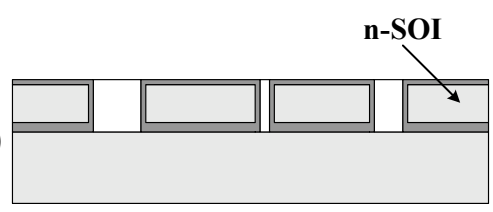

(B)

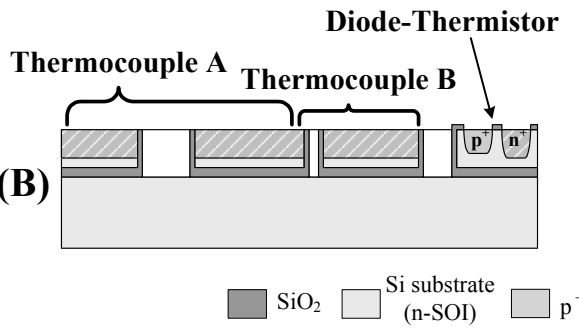

(C)

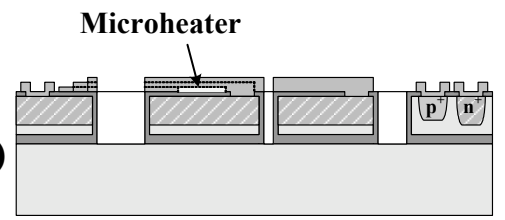

(D)

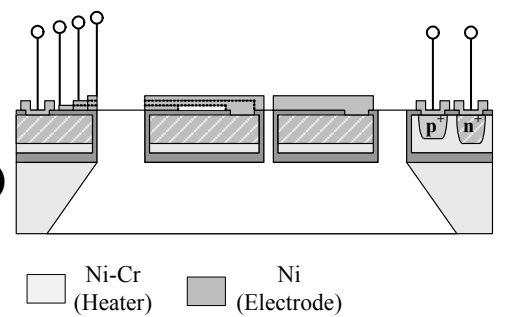

Fig.4: Fabrication process of the new type thin film vacuum sensor.

boron diffusion source of the OCD at $1100^{\circ} \mathrm{C}$ for 1 hour (Fig.4, step 2). The thickness of $\mathrm{n}^{++}$-Si diffused layer (assuming an average impurity concentration of about $1 \times 10^{20} \mathrm{~cm}^{-3}$ in this layer) is estimated to be about $1 \mu \mathrm{m}$ thick. The Ti/Nichrome/Ni metal (total thickness: about $0.3 \mu \mathrm{m}$ ) of a pair material in a single thermocouple is deposited by RF sputtering and patterned using the etching process (Fig.4, step 3). The thin film thermocouple is composed of the double layer of $\mathrm{n}^{++}-\mathrm{Si}$ and Ti/Nichrome/Ni metal film electrically isolated via the thermal $\mathrm{SiO}_{2}$ film (about $0.5 \mu \mathrm{m}$ thick) formed on the SOI layer as shown in Fig. 4(step 3 and step 4). The backside cavity to release the cantilever from the SOI substrate is formed using the anisotropic etchant of hydrazine for Si (Fig.4, step 4).

The thickness of the sputtering deposited Nichrome thin film of the microheater formed on the $\mathrm{SiO}_{2}$ layer on the SOI cantilever is controlled so as to be about $50 \Omega$, and the top deposited layer of the Ni film in the region of the microheater is etched away to increase its electrical resistance. The internal resistance $r$ of the fabricated thin film thermocouple in this experiment is about $57 \Omega$.

Slits are formed in the SOI cantilever in order to make thermal resistance both between the substrate and microheater, and between the regions $A$ and $B$.

\section{Measurements of Vacuum Pressure}

In Fig. 5 the measuring circuits for the new type thin film vacuum sensor using the short Seebeckcurrent detection type thermocouple is shown. 


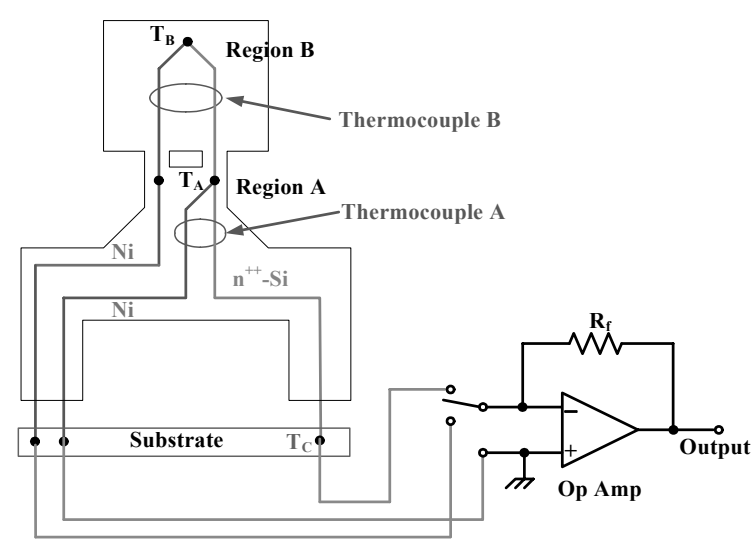

Fig.5: Schematic diagram of the measurement circuits for the proposed thin film vacuum sensor.

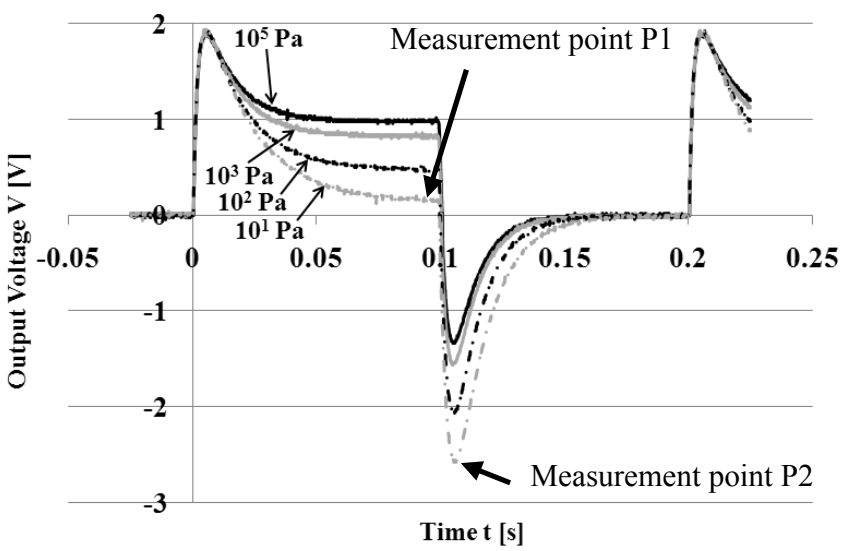

Fig.6: Output waveforms obtained from the thermocouple $B$ when microheater is driven as heating for $100 \mathrm{msec}$ and its cooling for $100 \mathrm{msec}$

Two thermocouples (thermocouple $A$ and thermocouple B) of a pair of the $\mathrm{n}^{++}-\mathrm{Si}$ layer and $\mathrm{Ti} / \mathrm{Nichrome} / \mathrm{Ni}$ metal film (mainly $\mathrm{Ni}$ thin film due to the low electrical resistance) are formed on the SOI cantilever. The thermocouple $\mathrm{A}$ can measure the temperature difference between the point in the region $A$, on where the microheater is formed, and the Si substrate, and the thermocouple $B$ can do between the region $A$ and the point in the region $B$ thermally isolated from the region $A$ by the slit. The cold junction and the hot junction of the thermocouple $B$ will be the junction formed in the region $B$ and the junction (region) formed in the region $\mathrm{A}$, respectively. The same $\mathrm{Ni}$ (Ti/Nichrome/Ni) metal film lead-line is extended from the Ohmic junction point with the $\mathrm{n}^{++}-\mathrm{Si}$ layer in the region $\mathrm{A}$ to the Si substrate rim to form the hot junction for the thermocouple B.

Temperature difference between the region $A$ and the thermally isolated region $B$ will be zero under the higher vacuum due to the cantilever structure. Therefore, the Seebeck current of the thermocouple B will be zero, namely the output voltage of the OP amplifier from the thermocouple B shown in Fig. 5 will also be zero under the high vacuum, such as $1 \times 10^{-4} \mathrm{~Pa}$.

The microheater is driven by constant current of about $40 \mathrm{~mA}$, and the increased temperature was about $190^{\circ} \mathrm{C}$ at this constant current under the high vacuum.

In our vacuum sensor, the output voltage has remained by only about $10 \mathrm{mV}$ for $\mathrm{Rf}=100 \mathrm{k} \Omega$ even under $1 \times 10^{-4} \mathrm{~Pa}$. Therefore, we have subtracted the remaining output voltage value from the data obtained from the vacuum pressure measurement.

Fig.6 shows output waveforms obtained from the thermocouple B when microheater is driven as heating for $100 \mathrm{msec}$ and its cooling for $100 \mathrm{msec}$. The vacuum pressure is measured at the measurement point P1 for lower pressure than about 0.5 atm. in Fig.6.

In Fig. 7 the linear scaled characteristics of the output voltage $V_{0}$ vs. the vacuum pressure $p$, measured at $\mathrm{P} 1$ in Fig.6, is shown.

In Fig. 8 the logarithmical scaled characteristics shown in Fig. 7 is shown.

We can see that we can measure the wide pressure range of about $2 \times 10^{-3}-1 \times 10^{5} \mathrm{~Pa}(1 \mathrm{~atm}$.) by use of our vacuum sensor with our newly developed Seebeck-current detection type thermocouples as shown in Fig. 7and in Fig. 8.

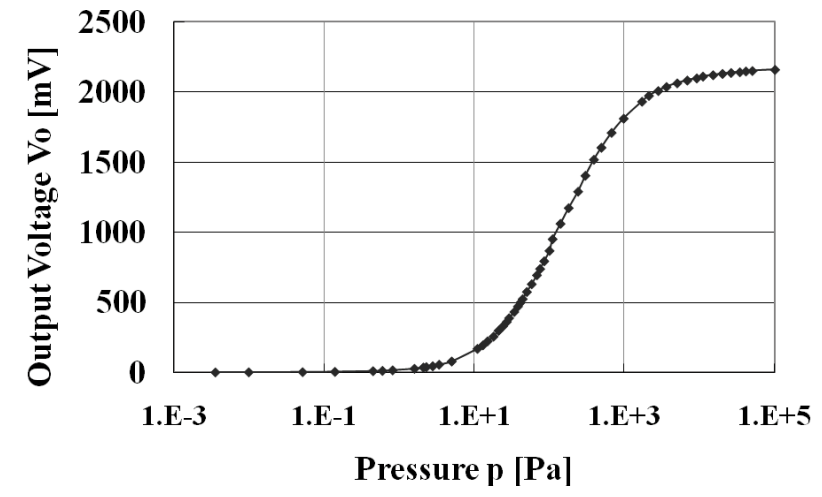

Fig.7: Linear scaled characteristics of the output voltage $V_{o}$ vs. the vacuum pressure $p$.

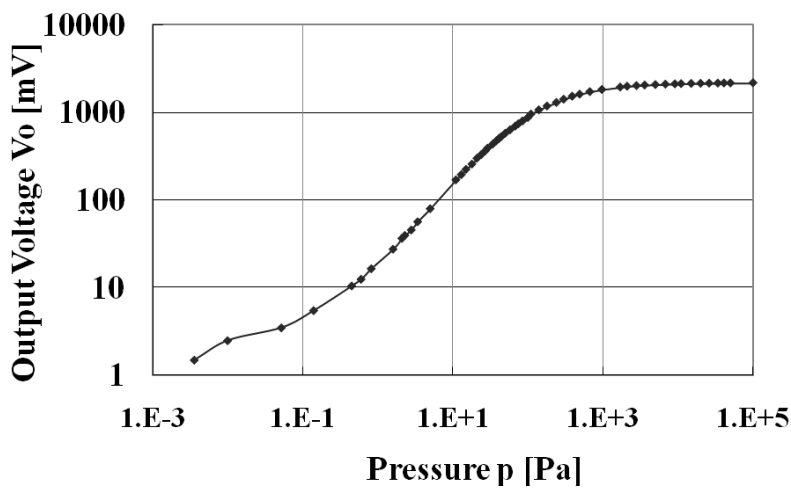

Fig.8: Logarithmical scaled characteristics of the output voltage $V_{o}$ vs. the vacuum pressure $p$. 


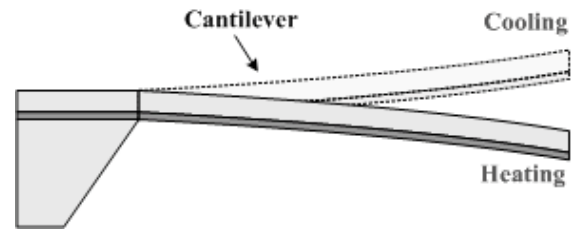

Fig.9: Schematic side view of the thin film Pirani vacuum sensor with vibration of the cantilever sensing region.

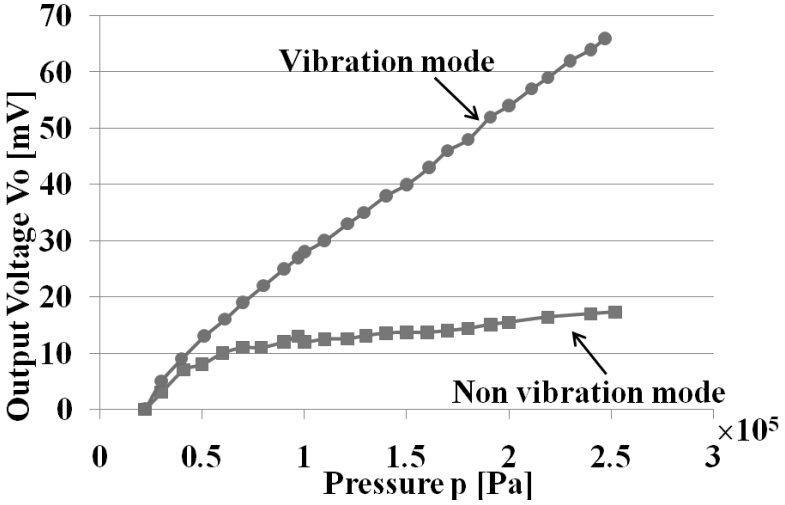

Fig.10: Improvement of the pressure sensitivity above $1 \mathrm{~atm}$. due to the vibration of the cantilever.

In Fig. 9 the schematic side view of the thin film Pirani vacuum sensor developed for high pressure measurement, in which the sensing cantilever is vibrating due to heating and cooling of the microheater, is shown.

This measurement method is based on the air convection cooling due to the vibration of the thin film cantilever composed of double layers of a SOI layer and a BOX $\left(\mathrm{SiO}_{2}\right)$ layer, on which a microheater and two temperature-difference sensors are formed. The cantilever will vibrate when it is heated up and cooled down based on its "on" and "off" state, like a bimetal thermal bending, because double layers of the cantilever have very large difference in their thermal expansion coefficients. In our experiments the microheater is driven so as to be heating for $15 \mathrm{msec}$ and cooling also for $20 \mathrm{msec}$ in order to repeatedly heat up to about $190^{\circ} \mathrm{C}$ of the saturated temperature at $100 \mathrm{~mW}$ under the higher pressure than near 1 atm. In this experiment with sensing-cantilever vibration the maximum amplitude of the output voltage Vo at $P 2$ shown in Fig .6, which is corresponding to the maximum temperature difference between the $A$ and the $B$ thermocouple, are measured after the microheater is turned off, because we can acquire the stable and reproducible pressure data due to vibration effects of the sensing cantilever.

In Fig.10 the output characteristics of the thin film Pirani vacuum sensor with the cyclic vibration mode and the non vibration mode are shown. We can see that the output voltage in high pressure range near 1 atm shows saturation characteristics as shown in Fig.7, however, as shown in Fig.10, increase in the output voltage $V o$ of the vibration mode is observed as increasing the pressure $p$, while the decrease in that of the non vibration mode is observed up to the pressure of $2.6 \mathrm{~atm}$. These effects with the vibration mode may be due to heat transfer by air convection through the surrounding high pressure air.

\section{CONCLUSIONS}

We have proposed the thin film vacuum sensor that has a cantilever structure with new temperature difference sensors of the short circuit Seebeck-current detection type thermocouple. In our experiments using the prototype sensor, very wide vacuum pressure range between $10^{-3} \mathrm{~Pa}$ and $10^{5} \mathrm{~Pa}$ can be reproducibly measured. We could expand the pressure sensitivity range from $1 \times 10^{5} \mathrm{~Pa}$ to $2.6 \times 10^{5} \mathrm{~Pa}$ by adoption of vibration of the sensing cantilever.

\section{REFERENCES}

[1] R. Puers, S. Reyntjens and D. De Bruyker, "The Nano Pirani-an extremely miniaturized pressure sensor fabricated by focused ion beam rapid prototyping", Sensors and Actuators A: Physical, Vol. 97-98, pp.208-214 (2002). [2] M. Kimura,"Microheater and microbolometer using microbridge of $\mathrm{SiO}_{2}$ film on silicon", Electron. Letters, Vol.17, No.2, pp.80-82 (1981).

[3] A. W. Van Herwaarden, P. M. Sarro and H. C. Meijer, "Integrated vacuum sensor", Sensors and Actuators,Vol. 8, No. 3 , pp.187-196 (1985).

[4] M. Kimura and K. Toshima," Thermistor-like pn junction temperature-Sensor with variable sensitivity and its combination with a micro air-bridge heater", Sensors and Actuators, A108, pp.239-243 (2003).

[5] Mitsuteru Kimura, Fumitoshi Sakurai, Hirao Ohta and Tomoyuki Terada," Proposal of A New Structural Thermal Vacuum Sensor with Diode-thermistors Combined with a Micro-Air-Bridge Heater", Microelectronics J. Vol.38, pp. 171-176 (2007).

[6] A. W. Van. Herwaarden, and P. M. Sarro, "Thermal sensors based on the Seebeck effect", Sensors and Actuators A Vol. 10, pp.321-346 (1986).

[7] Seung Seoup Lee, Mitsuteru Kimura," Short-circuit measurement by Seebeck current detection of a single thermocouple and its application", Sensors and Actuators, A139, pp.104-110 (2007). 